\title{
The Association of Clinical Pathologists: 96th general meeting
}

The 96th general meeting was held at the University of York on 8-9 April 1976. Abstracts of scientific communications follow. Three symposia were held, one on Countercurrent immunoelectrophoresis in bacteriology (Chairman: Dr R. J. Fallon), one on Standardization in clinical pathology (Chairman: Dr C. E. D. Taylor), and one on Malignancy in childhood (Chairman: Dr A. H. Cameron). Contributions to the last mentioned symposium are published on pages $1016-1037$ of this issue.

\section{Chromosomes and malignancy}

D. G. HARNDEN (Department of Cancer Research, University of Birmingham) Over the past 20 years many abnormalities of human chromosomes have been recognized and this has contributed a great deal to our knowledge of human disease. The chromosome abnormalities found in tumours are many and various but while their study has proved significant in understanding the nature of neoplasms, the practical clinical usefulness of cytogenetic studies on tumours is at present limited.

The occurrence of chromosome abnormalities is associated with an increased risk of developing cancer. This is shown in several different ways:

(1) Some patients with a constitutional chromosome abnormality have an unusually high risk of developing a malignant disease. For example, (i) patients with Down's syndrome (trisomy 21) have a heightened susceptibility to developing acute leukaemia; (ii) patients with Klinefelter's syndrome $(47, \mathrm{XXY})$ have an increased incidence of carcinoma of the breast as compared with normal males; (iii) females with a $46, \mathrm{XY}$ chromosome constitution have a high probability of developing dysgerminoma or gonadoblastoma.

(2) A group of diseases, often now called the chromosome breakage syndromes, have an increased cancer risk. Fanconi's pancytopenia patients tend to develop leukaemia or lymphoma. Cases of Bloom's syndrome and ataxia telangiectasia develop a variety of different malignancies but even in these instances the ie is a preponderance of lympoid neoplasms.

(3) The three environmental agencies which are clearly causes of cancer are all also chromosome damaging agents.
Patients known to have been exposed to ionizing radiation show chromosome damage for many years afterwards and also have an increased cancer risk. Human viruses which cause cancer in experimental animals and those (eg, EB virus) which are closely associated with human cancers cause extensive chromosome damage. The close association between carcinogenesis and mutagenesis is now clearly established, and most chemical carcinogens are chromosome damaging agents at levels much below the toxic dose.

So far, there is no firm evidence to link these chromosome changes, seen prior to malignant disease, with those observed in tumours but it seems probable that the variability which can be manifest as chromosome damage plays an important part in the progression towards full malignancy. It has been suggested that the chromosome abnormalities seen in cancers are themselves the primary change which causes malignancy but there is little evidence to support this idea and it seems better to regard these changes as evidence of a process of cellular evolution which is an on-going process in the dynamics of the tumour cell population.

Most of the chromosome changes that are seen in tumour cells involve changes of chromosome number. The actual number is often hypotetraploid but simple aneuploidy is not uncommon. Chromosome rearrangements occur, and within one tumour the chromosomes often clearly belong to a single clone (the stem line). Tumours of the same histological type usually have different chromosome abnormalities, but there are, however, some striking exceptions. In adult type chronic myeloid leukaemia a translocation between chromosome 9 and 22, leading to the production of a very small chromosome (the $\mathrm{Ph}^{1}$ or Philadelphia chromo- some), occurs in myeloid cells in over $90 \%$ of cases. It does not, however, occur in the juvenile type of CML. Some specificities are now emerging in the acute leukaemias (in particular, an excess of cases with trisomy of chromosome 8). In Burkitt's tumour there appears to be a specific translocation between chromosome 8 and 14 in the tumour cells. Meningioma cells are often hypodiploid, having specifically lost chromosome 22

The important clinical points are: (1) the presence of chromosome aberrations alone does not necessarily mean that malignant disease is already present but it could be an aid to other diagnostic tests; (2) the absence of chromosome abnormalities does not exclude malignancy because in some well-documented cases, especially leukaemias, the neoplastic cells have been shown to have normal chromosomes; (3) some specific changes (eg, the $\mathrm{Ph}^{1}$ chromosome) are of diagnostic value; (4) new specificities are now being discovered which may be of value in diagnosis in the future.

\section{References}

German, J. (1974). Chromosomes and Cancer. Wiley, New York.

Harnden, D. G. (1976). The cytogenetics of neoplasia. In Genetics and Human Cancer, edited by J. J. Mulvihill. Raven Press.

Adenosquamous carcinoma of the endometrium

M. T. HAQQANI AND H. FOX (Department of Pathology, University of Manchester) This work is published in full on pages 959-966 of this issue.

Exponential growth of medical laboratory work 1920-2000 AD

H. F. BARNARD (Westwood Hospital, 
Beverley) The records of this district are probably typical. Work was sent to Leeds and London until the Clinical Laboratory opened at Hull Royal Infirmary in 1922 and in Beverley in 1941. Before 1941 onefifth of the work is assumed to have been for Beverley District. Methods of recording have altered periodically. Runs of intrinsically comparable records were plotted on semilogarithmic paper and lined up. The load, throughout the 50 years, has grown exponentially, doubling every seven years. Extrapolated to 2000 $\mathrm{AD}$ work will multiply 12 times from the present amount. The staff and equipment at three points were allotted contemporary cost. The increase in cost will continue to be much less than the increase in load, and in relation to gross national product will be still less, perhaps being matched by a saving to the community and in bed use. Neither war nor slump seems to deviate the curve, and it will probably continue until someone delineates the work that is not needed.

A concomitant, county size specialization can be achieved without bigness and loss of local face-to-face contact by the specialization of each District Laboratory in certain categories of work.

Computer diagnosis elsewhere has already been shown to be advantageous to the patient and to achieve significant diagnosis with fewer investigations. If adopted, it will still be necessary to preserve a measure of test redundancy to allow for intuitive and random new finds and development.

Beyond this lies benefit budgeting. Functional budgeting presents departmental costs and seeks saving, seemingly by emulating low spenders and by the voluntary atrophy of departments. Barefoot medicine and sessions of self criticism are desirable aims. However, it is possible to evaluate the benefits to the community in cost and life quality and to put these beside the outlay. In suitable groups these can then be set in order of benefit. A simple start would surely be better than radomly doing good until cash runs out.

By such means can rationality hope to tame the dragon.

\section{The ACP Hepatitis Survey: a five-year study}

N. R. GRIST (Ruchill Hospital, Glasgow) Seventy-three cases of hepatitis (31 hepatitis B) were reported in laboratory staff for the years 1970-74, giving an average annual attack rate of $128 / 100000$. Attack rates at least twice the average were reported for medical staff (haematology 1970-72; morbid anatomy 1973-74), science graduate biochemists (1970-74), and technicians (biochemistry 1970-72; haematology 1973-74). Hepatitis was reported relatively most frequently from laboratories testing specimens from transplant units, often from those serving haemodialysis and haemophilia units or performing tests for HBsAg, and occasionally from those serving drug addiction centres. None of the hepatitis cases was fatal and only a quarter of them required hospital treatment ( $32 \%$ for hepatitis B, $22 \%$ for presumed hepatitis A). The sources of these infections of laboratory staff were usually uncertain, but contact with blood or with other persons with hepatitis or anicteric hepatitis B was reported more often for staff with hepatitis $\mathbf{B}$.

This continuing survey confirms the presence of some risk and the need for precautions but gives no grounds for alarm. Improvement seems called for in respect of graduate biochemists and haematology technicians.

Bacteriology of brain abscess: preliminary report of a collaborative study with neurosurgeons in England and Wales

J. DE LOUVOIS, ROSALINDE HURLEY, AND PETER GORTVAI (Queen Charlotte's Hospital for Women, London and Regional Centre for Neurosurgery, Romford, Essex) The mortality of brain abscess varies from 23 to $45 \%$ in published series, and from 9 to $63 \%$ are reported to be sterile. The high incidence of reported sterility has rendered ineffective attempts to improve the prognosis by reasoned use of antimicrobial drugs, although penicillin has been advised as the drug of choice.

We report a prospective study of 44 patients with intracranial (43) or intraspinal (1) abscesses. We conclude that: pus from abscess is not sterile; nontraumatic brain abscess is predominantly a streptococcal disease, and Streptococcus milleri is isolated most frequently; staphylococci predominate in post-traumatic abscess; and a large number of patients $(18 / 44)$ are infected by organisms that are resistant to penicillin.

\section{Otosclerosis and the pathologist}

INGLE WRIGHT (Department of Pathology, Stopford Building, Manchester, M13 9PT)
Understanding the material received from a stapedectomy can be very difficult, not least because the bony union of the footplate in the oval window may be so hard as to have been drilled. Three pieces of bone may arrive, and should be accompanied by the surgeon's description of what they are and whether the 'lesion' is present or not.

The commonest specimen has a footplate described as thick and white: the history is of many years' fixation; histologically the specimen is mainly lamellar bone. Earlier lesions, particularly in younger patients, may show great cellularity and the formation of new bone.

The head and crura may show osteoporosis; crypts of mucosal origin may penetrate the most porotic areas, and fat cells also may be seen.

Lipid-containing macrophage: are not uncommon in the stapedial niche and are irrelevant to the diagnosis.

Particular attention should be paid to the specimens removed early after the onset of deafness. These may contain patches or even large areas of avascular necrosis.

Discussion with the surgeon can be very fruitful for both: the surgeon's view at operation cannot be bettered in the laboratory, though it may be technically equalled with a Zeiss Stereo IV.

\section{Should blood films be examined?}

P. T. DILWORTH AND O. H. B. GYDE (East Birmingham Hospital, Birmingham) Routine blood counts and film examinations were analysed in order to determine whether the microscopy on all samples was worthwhile.

The factors taken into account were presence of details on the request form suggesting the need for microscopy, and whether all the results of the Coulter Model ' $S$ ' counter were within the normal range. Blood films were judged as normal or abnormal after conventional microscopic examination.

If request form clinical details and the Coulter counter results are ignored and no films are made, $26.6 \%$ of additional abnormalities will be missed. If films are made where indicated by the request form, the number of missed abnormalities falls to $15.5 \%$. By adding the samples with the counter results out of the normal range to the previous number having films examined, only $2.9 \%$ of unsuspected abnormalities are lost. Using the combined criteria, the number of films could be cut by $38 \%$. The time saved would be 
between 40 and 60 hours a month. The yardstick selected for microscopy will vary to suit local circumstances; the matter for concern will be the clinical importance of the small number of missed results. This survey yielded no immediate life-threatening problems. Pathological variation in numbers of the white cell series were found that were insufficient to alter the total count. Apparent abnormal platelet counts were noted, as were a number of morphological changes affecting all cell types. The development of an automated red and white cell sizing apparatus has proved useful but difficulties remain with platelets, eosinophils, and monocytes.

\section{Serotypes of meningococci}

D. M. JONES (Withington Hospital, Manchester) Group B meningococci are associated with a major proportion of meningococcal infections in the United Kingdom. In 1972 Frasch and Chapman (Infect. and Immun., 5, 98) described serotypes within the group B meningococci, and this report is of the serotypes prevalent among the strains isolated in the UK. Group B meningococci for this study were collected from three sources; from sporadic cases of meningococcal infection, from community outbreaks of infection, and from patients who were nasopharyngeal carriers and had no association with any clinical meningococcal disease. The results show that the serotype 2 antigen is especially common among strains associated with both epidemic and sporadic clinical disease and that this serotype is relatively uncommon among strains carried by the general population. Meningococcal infections due to the group W135 have been reported with increasing frequency in the UK during the last two years. Serotyping strains of this group isolated from clinical cases also shows that a majority have the serotype 2 antigen. The significance of these findings will be discussed.

Report on a clinical trial of Tibric acid, a new hypolipidemic agent

J. B. ENTICKNAP, R. S. WINWOOD, AND P. L. WRIGHT (Department of Pathology, Whipps Cross Hospital, London E11) 2-Chloro(3,5-dimethylpiperidinosulphono) benzoic acid (Pfizer compound 18 524) is an anti-inflammatory drug that was found to have a hypolipidemic action in rats. Trials have been conducted to assess the efficacy and safety of the drug and to confirm by simple clinical chemistry the supposed mode of action. As a preliminary to a full clinical trial we have tested eight elderly volunteers with type IIb (VI) hyperlipidemia.

In the preliminary trial blood levels of free glycerol fell sharply and were consistent with the hypothesis that Tibric acid induces activity of mitochondrial $\alpha$ GPDH.

Forty-seven subjects were randomly allocated to a test group, a placebo group, and a group treated with clofibrate. Both drugs produced significant falls in blood levels of cholesterol and triglyceride which were maintained for up to six months. There seemed to be little to choose between them.

\section{Using the figures from the Coulter-S counter}

G. A. C. SUMmers (County Hospital, York, Y03 $7 P G)$ The haematologist may control the requests made to his laboratory if he can control the relevance of his reports. Assuming he does not manage clinically every case he reports on, his best hope of preventing unnecessary tests lies in maintaining an up-to-the-minute record of everything going on around each patient and especially those, perhaps designated as mere 'counts', in whom he may not be expected to take particular interest. Such a record can be maintained by expensive computers under full laboratory control, with a close ward link and frequent contact with the general practitioners. But, however speedily a computer may work, the pattern of its visual or paper display still has to be scanned. As this scanning must take time it is usually done on the haematologist's selected cases only.

Although a clinical case-pattern can often be divined from a patient's identity, age, and clinical source (consultant, houseofficer or practitioner), it frequently proves impossible to make this selection on this information even with a helpful clinical note.

Coulter-S automation saves space, speeds figures, and improves accuracy but usually increases demand. However, it has been shown in York that, if consistent attention is paid to relevance in reporting, and if all parts of each report are sent out together on the same day as the request, the workload does not increase. With a smaller mass of daily data to sift through before filing the lab-record ready for tomorrow, more time is available for considering selected blood-cases. A rigid lab-recording system, which can be operated manually, makes double-checking quick and easy. Locally, this activity is called 'policing' because it carries the threat of discussion of any error found; but this is no more serious than the possibility of re-examination of controversial bloodfilms, which is also readily done.

The form and order of the report is dictated by local custom and, if adhered to, it aids clinical appreciation of its pattern and significance. An object of this paper is to show how this pattern may be kept visible without having to turn up reports to see those hidden underneath. The same principle of order on the lab-record enables changes in the case-progress pattern to be shown in almost graphic manner even in the most trivial seeming situations. Preparation for a computer's mark-sensor or for microfilming of records is thus made.

In York the problem of superimposing the often irrelevant figures produced by the Coulter-S counter was not immediately overcome. The present compromise, effected to maintain the coincidence of reporting figures, bloodfilm survey, and consideration of previous and other related reports' relevance, involves omission of the red cell count and of the mean cell-haemoglobin concentration (used only as technical quality-control within thelaboratory). The packed cell volume is also omitted, the formula $\mathrm{PCV}=\frac{\mathrm{MCV}}{\mathrm{MCH}} \times \mathrm{Hb}$ being presented to encourage reliance on the haemoglobin level every time there is anxiety about relative red cell volume. Not only does this MCV/ MCH ratio usually work out at around 3, but it becomes quite useful in identity checking owing to its constancy in a stable situation in the individual's blood picture. Its pattern (without doing any mental arithmetic) proves an easy guide to progress as well as a case-screening aid and is, I believe, the most helpful aid since the discovery over a quarter of a century ago of the need to make a bloodfilm with every count requested.

Graphs of workload and diseaseincidence recorded, with the screening and lab-record system as it has developed, are shown along with detail of record-method and abbreviations locally used.

\section{Anaerobic bacteriology in York}

R. N. PEEL (County Hospital, York) Recent interest in anaerobic bacteriology 
has led to the establishment of methods for isolating and identifying exacting anaerobic bacteria from clinical sources, mainly surgical wound infections. The collection of specimens, their culture, and the use of such devices as an anaerobic glove box is discussed. A scheme for identification of anaerobic bacteria using simple tests and gas-liquid chromatography is presented and the results are summarized.

Aminoglycoside antibiotics and renal function: changes in urinary gammaglutamyl transferase excretion

P. R. BECK AND A. K. R. CHAUDHURI (Department of Biochemistry, Royal Infirmary, Glasgow and Department of Infectious Diseases, Belvidere Hospital, Glasgow) Urinary excretion of the renal proximal tubularenzyme, $\gamma$-glutamyltransferase $(\gamma-G T)$, has been studied in patients treated with the aminoglycoside antibiotics tobramycin, gentamicin, and streptomycin. Urinary $\gamma$-GT excretion was expressed as $U / 24 h$ and as a ratio to creatinine clearance, the latter being a better index of changes in urinary $\gamma-\mathrm{GT}$ output.

All patients treated with tobramycin or gentamicin for a variety of infections showed increased urinary $\gamma$-GT excretions. The increases ranged from 1.5 to 19 times the upper limit of normal, and these antibiotics seemed equally potent in their effects on enzyme excretion. Serial studies in many of the patients showed that the increased enzyme excretion became apparent one to four days after starting antibiotic therapy and it returned to normal three to 45 days after stopping therapy. Other tests of renal function (plasma urea and creatinine, creatinine clearance, and urinary protein) did not change during treatment. The degree of increase of $\gamma$-GT above normal was inversely related to the initial creatinine clearance.

Only $50 \%$ of patients treated with streptomycin for tuberculosis showed increases in urinary $\gamma-\mathrm{GT}$, and these increases have ranged from 1.2 to 1.8 times normal.

One patient treated with kanamycin has also shown increased urinary $\gamma$-GT activities.

Although it is clear that the aminoglycoside antibiotics are capable of affecting the kidneys, this does not affect the 'classical' renal function tests and therefore need cause little anxiety for the patient's safety. On the other hand, patients with pre-existing renal impairment are most likely to respond unfavourably to administration of these antibiotics.

Action of mecillinam upon Gram-positive urinary tract pathogens under conditions simulating urinary tract infection

J. D. ANDERSON, MONICA A. ADAMS, C. A. SHEPHERD, AND L. C. WILSON (Infection Control Laboratory, Clifton Hospital, York Y03 6RD) Pivmecillinam is an ester of a new penicillin analogue which is hydrolysed after absorption by non-specific esterases to release mecillinam.

The Micrococcaceae appear to be very resistant to mecillinam by the conventional antibiotic sensitivity test with paper discs containing $10 \mu \mathrm{g}$ of antibiotic. However, the minimum inhibitory concentrations of mecillinam for 35 urinary isolates of Micrococcaceae were found to be much lower than urinary concentrations, and urine from volunteers receiving therapeutic doses of pivmecillinam was cidal for almost all isolates. The effect of a range of concentrations of added mecillinam upon the growth of 10 novobiocin-resistant subgroup 3 micrococci (Staphylococcus saprophyticus, biotype 3) was studied in urine. Mecillinam was bacteriostatic or bactericidal for all 10 isolates at a concentration of about one quarter of the mean value found in the urine of patients receiving standard doses of pivmecillinam. These experiments, and evidence that urinary rather than serum antibacterial activity determines the outcome of therapy, suggest that uncomplicated urinary tract infections by Micrococcaceae may be expected to respond to treatment with pivmecillinam. A disc containing $\mathbf{5 0}$ $\mu \mathrm{g}$ of mecillinam may more accurately predict the outcome of treatment than the $10 \mu \mathrm{g}$ disc previously used.

Similar studies indicate that urinary infections by faecal streptococci are unlikely to respond to standard doses of pivmecillinam.

\section{Pulmonary amyloidosis: a case report}

D. J. GEE, S. M. RAJAH, AND D. J. CHARLEY (Department of Forensic Medicine, University of Leeds) A 60-year-old man suffered from chronic chest trouble for which he had been seen at the local chest clinic. Haematological investigation showed considerably increased amounts of immunoglobulins and a diagnosis of monoclonal gammopathy was made. He was treated with prednisone but died a few months later following a haemoptysis. His death was reported to the coroner.

At postmortem examination his lungs were seen to be studded with large numbers of nodules, several centimetres in diameter which, from external examination, suggested multiple secondary deposits of tumour; on the cut surface the tissues contained multiple, large, rounded, gelatinous semitranslucent nodules up to a centimetre or so in diameter scattered throughout the lungs. These nodules tended to shell out, leaving small cavities in the lung tissue. No microbes were isolated. Microscopical examination showed large masses of amyloid in the lung.

Postoperative chest infection: a laboratory and clinical assessment

P. J. WILKINSON, A. J. BALL, J. DORAN, VIVIAN S. ORTON, AND W. A. GILLESPIE (Departments of Clinical Bacteriology and Surgery, Bristol Royal Infirmary, Bristol $B S 28 H W$ ) Patients admitted for elective abdominal operations were examined regularly for signs of lower respiratory infection. Samples of their sputum were investigated in the absence of clinical information by grading purulence and examining a Gram-stained smear and culture. Clinicoradiological evidence of lower respiratory infection was correlated retrospectively with the laboratory findings. The significance of various organisms as respiratory pathogens and the effects of antibiotic treatment on the sputum flora were assessed. The value of selective and non-selective culture methods and of liquefaction of the sputum was also determined.

Clinically severe infections were often associated with a predominant growth of Pneumococcus and/or Haemophilus from the sputum. Heavy growth of other Gramnegative bacilli (coliforms etc) and of Staphylococcus aureus was less clearly associated with clinical features of infection and often followed antibiotic therapy. Liquefaction methods in examining sputum had no clear advantage when both selective and non-selective media were used.

Variability in the spectrum of activity of $\beta$-lactamase preparations

S. SELWYN (Westminster Medical School, London) Commercially produced 'penicillinases' which are recommended for 
routine use in clinical bacteriology (Waterworth, 1973) have been found to vary widely in their activity and to be uniformly ineffective against a recently introduced cephalosporin, cephradine. Conventional enzyme assays, based on either microbiological or chemical pro¿ cedures, involve the use of multiple sets of tube or plate tests if they are to cover the full range of lactam antibiotics. A simplified test procedure has therefore been devised to allow the rapid screening of batches of enzymes for their potency against the entire range of penicillins and cephalosporins in clinical use. The method requires only one agar plate for each enzyme preparation. A fully sensitive bacterial strain is inoculated over the entire surface and then a concentrated solution of enzyme is spread in two narrow parallel bands $4 \mathrm{~cm}$ apart. Finally, a row of standard antibiotic discs is placed tangentially to each enzyme band. The same procedure can readily be used to obtain detailed information on the spectrum of action of any $\beta$-lactamase produced by a bacterial isolate if the organism is inoculated in place of the prepared enzyme.

Using this method only one $\beta$-lactamase preparation-derived from Enterobacter cloacae-possessed adequate activity against cephradine and the slightly less enzyme-resistant antibiotic, cephalexin. This preparation is not commercially available, but the use of an enzyme of this type is essential if antibiotic inactivation of cephalosporins is required in blood cultures, mixed antibiotic assays or sterility tests.

\section{Reference}

Waterworth, Pamela M. (1973). An enzyme preparation inactivating all penicillins and cephalosporins. J. clin. Path., 26, 596-598.

Errors in viable counts due to bacterial aggregation

RAJAA M. MILYANI AND S. SELWYN (Westminster Medical School, London) Viable counts of bacteria are becoming increasingly important in clinical microbiology. Apart from their long established use in testing samples of food, water, and milk, and in standardizing the inoculum size in antibiotic sensitivity tests and assays, procedures for quantitative culture are playing a progressively greater part in the investigation of clinical samples such as urine, sputum, and blood. The validity of all these applications rests on the basic premise that when a bacterial suspension is suitably diluted each viable bacterium will give rise to a single discrete colony. But this is, of course, an erroneous assumption since the cells of most bacterial species tend to form aggregates of varying sizes, and consequently the estimated viable count may be considerably lower than the true number of living organisms in any sample. On the other hand, however, not all the cells in an aggregate can be assumed to be viable.

The extent of the practical problem was determined by calculating the average number of cells per aggregate as well as the conventional viable and total counts in strains of a wide range of species grown in broth and sterile urine. Parallel experiments were performed using mechanical and chemical means in an attempt to reduce the size of the bacterial aggregates. These are, however, surprisingly stable, and a considerable decrease in their size was obtained only in the case of Streptococcus pyogenes where the average number of cells was reduced from 25 per aggregate to 5 by continuous shaking of the incubated cultures. Wide variations in aggregate size were found among different strains of each species, therefore correction factors cannot be applied rigidly. Average factors have, however, been calculated and should prove useful in practice. They range from 15 for Strep. pyogenes to 2 for Klebsiella aerogenes. Further correction factors have been calculated to compensate for the presence of varying proportions of non-viable cells under different conditions.

Water soluble vitamin excretion in duodenal ulceration

J. W. M. KEeN AND A. G. GREen (County Hospital, York) Over a period of two years, 362 24-hour specimens of urine were collected from groups of controls, patients with duodenal ulcers, and patients who had undergone five types of gastric operation. Specimens were analysed for creatinine and three watersoluble vitamins - pantothenic acid, thiamine, and N'methylnicotinamide (NMN).

The results showed:

(1) a significant reduction in the excretion of creatinine, thiamine, and NMN after all five types of gastric operation;

(2) a significant reduction in the excretion of pantothenic acid after all types of operation except vagotomy and pyloroplasty;

(3) a significantly reduced excretion of creatinine, NMN, and thiamine in patients with duodenal ulcers before operation.

Blood thiamine levels were estimated in controls and partial gastrectomy patients. The results showed that there was a significantly lower level of blood thiamine compared to controls, indicating that the reduced urinary excretion reflected a lower blood level for this vitamin.

The results of the assays of watersoluble vitamins in urine suggested that duodenal ulcer patients could suffer from subclinical deficiencies of several vitamins of the B complex both before and after operation. Enquiries into the dietary habits of patients indicated that, in general, the food intake was not sufficiently affected to account fully for the changes in urinary excretion. Similarly, differences in weight could not account fully for the reduction in urinary creatinine output in all groups studied.

A seasonal variation was demonstrated in NMN excretion in controls and partial gastrectomy patients.

\section{Whole blood viscosity studies in myeloma- tosis}

F. E. PRESTON, R. B. COOKE, AND N. F. BURROWs (University Department of Haematology, Sheffield and Physical Biochemistry Department, Westminster Medical School, London) The hyperviscosity syndrome is a recognized complication of Waldenström's macroglobulinaemia but is considered rare in myelomatosis.

Attention has recently been drawn to the increased frequency of this syndrome in IgA myelomatosis. In order to investigate this further we have performed whole blood viscosity (WBV) measurements on blood samples obtained from patients with myelomatosis and macroglobulinaemia. Blood viscosity was measured at two rates of shear using a cone and plate microviscometer. Results obtained show that for a given increase in paraprotein concentration there is a much greater rise in WBV in samples from IgA myeloma compared with IgG myeloma. Ultracentrifugation analysis revealed high molecular weight complexes in plasma samples from patients with IgA myelomatosis. There was no correlation between WBV and 7S protein but significant correlation was demonstrated between $\mathrm{WBV}$ and $10+12 \mathrm{~S}$ protein. The increased frequency of hyperviscosity syndrome in IgA myloma is almost certainly due to the 
formation of high molecular weight complexes in the patient's plasma.

\section{Disseminated intravascular coagulation and intestinal disease}

F. P. RYAN, F. E. PRESTON, W. R. TIMPERLEY, AND C. D. HOLDSWORTH (Departments of Medicine, Haematology and Neuropathology, Royal Infirmary, Sheffield) Seven patients are described in whom disseminated intravascular coagulation (DIC) occurred as a serious complication of intestinal disease. Four patients had Crohn's disease, two had ulcerative colitis, and one had coeliac disease. Unequivocal haematological evidence of DIC was obtained in all seven patients, and this was associated, in six patients, with neurological manifestations.

Five patients died, and histological confirmation of the intravascular coagulation was obtained in the four patients on whom necropsy was performed. Of particular interest was the demonstration of microthrombi in the intracerebral vessels of three of the patients.

In view of a previous report (Ryan et al, 1974), the authors believe that in the patients with ulcerative colitis hyposplenism may have been an important factor leading to increased susceptibility to DIC. One patient with Crohn's disease developed a florid DIC syndrome without infection and the trigger was considered to be the inflammatory disease of the bowel. Infection was the probable trigger in the other three cases.

When treatment with heparin was delayed the DIC progressed and was a significant cause of death. Early diagnosis and treatment with heparin satisfactorily controlled the DIC in the two patients who survived.

\section{Reference}

Ryan, F. P., Smart, R. C., Preston, F. E., and Holdsworth, C. D. (1974). Hyposlenism in ulcerative colitis. Lancet, 2, 318.

\section{Use of tests of fetal-placental function}

D. R. C. WILlcox (Princess Alexandra Hospital, Harlow, Essex) Urinary oestriol and serum placental lactogen (HPL) were assayed concurrently during the latter part of 400 pregnancies. The reasons for the investigations and the frequency of testing were at the discretion of the obstetrician. The subjects included a group with normal pregnancy but mainly with previous bad obstetric histories (63), elderly patients (33), a group with 'static weight' (94), a group 'small for dates' (49), pre-eclamptic toxaemia (PET) (82), and other smaller groups.

Abnormally low values were found in 45 patients $(11 \%)$; in 18 only oestriol was low, in 20 only HPL was low, and in seven both were low. These represented $20 \%$ of PET patients, $12 \%$ of the smal for dates group, and $10 \%$ of the static weight group, compared with $5 \%$ of the normal group. Few of these results, however, appeared to influence clinical management, and a review of case-notes revealed only eight patients (1 normal, 2 small for dates, 5 PET) in whom the results had contributed to a clinical decision, which was usually to induce labour.

On the other hand, some pregnancies showing normal values for both tests were associated with fetal and neonatal problems. To assess the predictive value of the tests cases were regrouped as fetal and neonatal deaths (8), low birth weight infants (31), and neonate admissions to the special care unit (25). Less than $40 \%$ of mothers in each group had shown any abnormal values.

It is concluded that these tests are of limited and rather occasional value either for immediate management or for prediction of fetal and neonatal difficulties. Their present routine use is questioned and there is need to define indications for their use as well as to clarify their significance.
Biochemical monitoring in myeloproliferative disorders

B. E. ROBERTS, E. H. COOPER, R. TURNER, AND J. A. CHILD (Department of Haematology, The General Infirmary at Leeds and Department of Cancer Research, The University of Leeds) Regular biochemical monitoring of patients with myeloproliferative disorders has been performed over a period of one to two years in an attempt to predict changes in the course of the disease and assess the effect of the disease and therapy on the liver.

Persistent disturbance of liver function in polycythaemia vera can occur at all stages of the disease. Gamma glutamyl transpeptidase (GGT) is the most sensitive indicator; 42/68 were within normal limits, $17 / 68$ were between $30-60 \mathrm{IU} / 1$, and $9 / 68$ were greater than 61 IU/1. Treated congestive heart failure complicating polycythaemia vera was associated with an elevation of GGT in 8/10 patients. Alanine aminotransferase levels were raised in 2/61 and aspartate aminotransferase in 10/61. A moderate increase of bilirubin was observed in $32 \%$ but this appears to be independent of hepatic parenchymal damage. The relation of this change to haptoglobin, haemopexin, and lactic dehydrogenase levels is presented. The serum lysozyme index shows three patterns: high indices associated with low granulocyte counts, low indices with high granulocyte counts, and cyclical variation independent of therapy.

Similar tests were performed on patients with chronic myeloid leukaemia. Evidence of chronic hepatic parenchymal damage was found in $8 / 19$ cases, but, with two exceptions, the rate of progression appeared to be very low. The haptoglobin levels were variable and could be consistently very high (greater than $2.0 \mathrm{~g} / \mathrm{l}$ ) or very low (less than $0.6 \mathrm{~g} / \mathrm{l}$ ), during the course of a year. The lysozyme index tended to remain low despite the high total leucocyte count and can vary independently of the granulocyte or total leucocyte count. 